\title{
Base Point Free Pencils on Multiple Coverings of Smooth Curves
}

\author{
E. Ballico ${ }^{1}$ \\ Dept. of Mathematics \\ University of Trento \\ 38050 Povo (TN), Italy \\ ballico@science.unitn.it
}

\begin{abstract}
Fix an integer $k \geq 2$ and a smooth genus $q$ projective curve $X$. Here we prove the existence of a smooth curve $C$, a degree $k$ morphism $C \rightarrow X$ and a low degree base point free linear system on $C$ not coming from $X$.
\end{abstract}

\section{Mathematics Subject Classification: 14H51; 14H30}

Keywords: multiple coverings of curves; base point free pencils

We will follow the notations of [2] and [3] concerning ruled surfaces over a smooth projective curve $X$. If $S:=\mathbf{P}\left(\mathcal{O}_{X} \oplus R\right), R \in \operatorname{Pic}(X), \operatorname{deg}(R) \leq 0$, we will write $X_{0}$ (resp. $X_{1}$ ) for the unisecant divisor of the ruling $S \rightarrow X$ associated to the projection of $\mathcal{O}_{X} \oplus R$ onto the first (resp. second) factors. Thus $X_{0}^{2}=\operatorname{deg}(R), X_{1}^{2}=-\operatorname{deg}(R)$ and $X_{0} \cap X_{1}=\emptyset$. The case $k=2$ of the following lemma is just [1], Lemma 2.2. Unless otherwise stated $X$ is a smooth and connected projective curve of genus $q$ and $S:=\mathbf{P}\left(\mathcal{O}_{C} \oplus R\right), R \in \operatorname{Pic}(X)$, $e:=\operatorname{deg}(R) \leq 0$. For any complete linear system $|a|$ on $X$, let $\operatorname{Bs}(|a|)$ denote its base locus.

Here we follow the proofs of [1] and prove the following results.

Theorem 1. Let $X$ be a smooth curve of genus $q$. Fix an integer $k \geq 2$ and effective divisors $a_{1}, a_{2}, b$ on $X$ such that $b$ and $b+k a_{2}-k a_{1}$ are smooth and with disjoint support, $b+k a_{2}-k a_{1} \neq \emptyset$ and $B s\left(\left|a_{1}\right|\right) \cap B s\left(\left|a_{2}\right|\right)=B s\left(\left|a_{1}\right|\right) \cap B s(|b|)=$ $B s\left(\left|a_{2}\right|\right) \cap B s\left(\left|b+k a_{2}-a_{1}\right|\right)=\emptyset$. Set $R:=\mathcal{O}_{X}\left(a_{2}-a_{1}\right), e:=-\operatorname{deg}(R)=$ $\operatorname{deg}\left(a_{1}\right)-\operatorname{deg}\left(a_{2}\right)$, and $S:=\mathbf{P}\left(\mathcal{O}_{C} \oplus R\right)$. Fix a general $C \in\left|k X_{0}+b f\right|$ and $W$ be the the complete linear system on $C$ associated to $\left(X_{0}+a_{1} f\right)_{\mid C} . C$ is smooth and irreducible, $p_{a}(C)=1+k(q-1)+(-k(k-2) e+(k-2) \cdot \operatorname{deg}(b)+$

\footnotetext{
${ }^{1}$ The author was partially supported by MIUR and GNSAGA of INdAM (Italy).
} 
$\left.k \cdot \operatorname{deg}\left(b+a_{2}-a_{1}\right)\right) / 2$, the linear system $W$ has no base points and degree $-k e+\operatorname{deg}(b)+k \cdot \operatorname{deg}\left(a_{1}\right)$.

Theorem 2. Fix positive integers $q, k, d$, such that $k \geq 2$. Set $g:=1+k(q-$ $1)+(-k(k-2) e+(k-2) \cdot \operatorname{deg}(b)+k \cdot \operatorname{deg}(b)) / 2$. Let $X$ be a smooth curve of genus $q$ such that there is a base point free degree $d$ pencil. Then there are a smooth genus $g$ curve $C$, a degree $k$ covering $u: C \rightarrow X$ and a degree $d$ base point free linear system on $C$ not induced from a linear system on $X$.

Lemma 1. Fix an integer $k \geq 2$. Let $b_{1}, b_{2}$ be smooth effective divisors on $C$ with disjoint support and such that $\mathcal{O}_{X}\left(b_{2}-b_{1}\right) \cong R^{\otimes k}$. If $R \cong \mathcal{O}_{X}$, then assume $b_{1} \neq \emptyset$. Then there exists a smooth and irreducible curve $C \in \mid k X_{0}+$ $b_{1} f \mid$.

Proof. Let $C \in\left|k X_{0}+b_{1} f\right|$ be a general element. Let $B$ be the set-theoretic base locus of the linear system $\left|k X_{0}+b_{1} f\right|$. Notice that $k X_{1}+b_{2} f \in\left|k X_{0}+b_{1} f\right|$. Since $b_{1} \cap b_{2}=X_{0} \cap X_{1}=\emptyset, B$ is finite. By Bertini's theorem to check that $C$ is smooth it is sufficient to show that $C$ is smooth at each point of $B$. Since $b_{1} \cap b_{2}=X_{0} \cap X_{1}=\emptyset$ and $k X_{1}+b_{2} f \in\left|k X_{0}+b_{1} f\right|, B$ is contained in $\left(b_{1} f \cap X_{1}\right) \cup\left(b_{2} f \cap X_{0}\right)$. At each of these points $C$ is smooth, because $b_{1}, b_{2}$ are smooth. Now we check the irreducibility of $C$. Since $C$ is irreducible, it is sufficient to show that $C$ is connected. Since $k X_{1}+b_{2} f \in\left|k X_{0}+b_{1} f\right|$, $b_{1} \cap b_{2}=X_{0} \cap X_{1}=\emptyset$ and $C$ is general, neither $X_{0}$ nor $X_{1}$ nor any fiber of the ruling is a connected component of $C$. Assume $C=A \cup B$ with $A \neq \emptyset, B \neq \emptyset$ and $A \in\left|x X_{0}+y f\right|, 0<x<k$. Since $X_{0}$ is not a component of $C$, we get $X_{0} \cdot A \geq 0$ (i.e. $\left.\operatorname{deg}(y) \geq e x\right)$ and $X_{0} \cdot B \geq 0$ (i.e. $\operatorname{deg}\left(b_{1}\right)-\operatorname{deg}(y) \geq e(k-x)$ ). We get $A \cdot B \geq 0$ with equality if and only if $e=0$ and $\operatorname{deg}\left(b_{1}\right)=0$ (i.e. $b_{1}=\emptyset$ ). Our assumpotions imply $A \cdot B>0$. Hence $A \cap B \neq \emptyset$, contradiction.

Proof of Theorem 1. Apply Lemma 1 with $R=\mathcal{O}_{X}\left(a_{2}-a_{1}\right), b_{1}=b$ and $b_{2}=b+k a_{2}-k a_{1}$. We get that $C$ is smooth and irreducible. Notice that $\omega_{S} \cong$ $\mathcal{O}_{S}\left(-2 X_{0}+\left(\omega_{C}+R\right) f\right)$. Hence $\omega_{C} \cong\left((k-2) X_{0}+\left(\omega_{C}+R+b\right) f\right)_{\mid C}$ (adjunction formula). Hence $2 p_{a}(C)-2=\left(k X_{0}+b f\right) \cdot\left((k-2) X_{0}+\left(\omega_{C}+R+b\right) f\right)$. Since $X_{1}+a_{2} f \in\left|X_{0}+a_{1} f\right|$, the base points of $\left|X_{0}+a_{1} f\right|$ are contained in the finite set $\left(X_{0} \cap a_{2} f\right) \cup\left(X_{1} \cap a_{1} f\right)$. Since $C \in\left|k X_{0}+b f\right|, C \cap X_{0}=\left(b_{2}+k a_{2}-k a_{1}\right) f \cap X_{0}$ and $C \cap X_{1}=b f \cap X_{1}$. Since $\operatorname{Bs}\left(\left|a_{2}\right|\right) \cap \operatorname{Bs}\left(\left|b+k a_{2}-a_{1}\right|\right)=\emptyset$, and $C$ is general, we get $C \cap X_{0} \cup a_{2} f=\emptyset$. Since $\operatorname{Bs}\left(\left|a_{1}\right|\right) \cap \operatorname{Bs}(|b|)=\emptyset$ and $C$ is general, $C \cap X_{1} \cap a_{1} f=\emptyset$. Hence $W$ has no base points.

Proof of Theorem 2. Apply Theorem 1 taking $a_{1}=a_{2}=0$.

We work over an algebraically closed field $\mathbb{K}$ such that $\operatorname{char}(\mathbb{K})=0$.

\section{REFERENCES}

[1] L. Fuentes García, Pencils on double coverings of curves, arXiv:math.AG/0701472.

[2] L. Fuentes García and M. Pedreira, The projective theory of ruled surfaces, Note Mat. 24 (2005), no. 1, 25-63. 
[3] L. Fuentes García and M. Pedreira, Canonical geometrically ruled surfaces, Math. Nachr. 278 (2005), no. 3, 240-257.

\section{Received: January 29, 2007}

